\title{
Protée
}

\section{De dialogues et de paradoxes}

\section{Claudie Gagné}

Volume 27, numéro 2, 1999

\section{La réception}

URI : https://id.erudit.org/iderudit/030555ar

DOI : https://doi.org/10.7202/030555ar

Aller au sommaire du numéro

Éditeur(s)

Département des arts et lettres - Université du Québec à Chicoutimi

ISSN

0300-3523 (imprimé)

1708-2307 (numérique)

Découvrir la revue

Citer cet article

Gagné, C. (1999). De dialogues et de paradoxes. Protée, 27(2), 15-22.

https://doi.org/10.7202/030555ar

\section{Résumé de l'article}

La présente réflexion explore le seuil critique du rapport spéculaire de l'objet et du sujet qui se croisent et qui, littéralement, se traversent l'un l'autre par la médiation du langage artistique. La spécularité qui en résulte est

l'interlocution en acte avec l'autre, ici l'objet littéraire qui se présente à soi de la même manière qu'on tend à l'aborder, ayant en partage et pour point de départ les caractéristiques du pluriel et de la privation déposées au coeur du langage ; terrain de la rencontre esthétique et occasion aussi, sur une autre échelle, de susciter une expérience dynamique entre un art bigarré - porteur d'un questionnement sur le monde dont il est issu - et le regard critique qu'il informe. 


\section{DEDIALO GUESET DE PARADOXES}
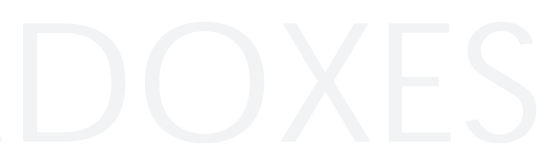

Claudie Gagné

Peut-être faudrait-il chercher à édifier quelque chose de l'ordre d'une épistémologie affective qui se donnerait pour objet d'étude l'usage que nous faisons de nos théories dans le travail quotidien et les rapports compliqués, largement irrationnels que nous entretenons avec cet objet mental qu'est la théorie.

J. Hochmann, 1988: 120

Jacques Geninasca, dans une livraison récente de Protée, dit vouloir opposer, à une interprétation de spécialiste,

[...] une réflexion herméneutique qui, s'interrogeant sur elle-même, s'efforce d'expliciter les présupposés ou d'imaginer les axiomes qui en assurent la possibilité. Tout faire interprétatif, comme toute lecture d'ailleurs, est tributaire d'une conception du langage, de la signification, du discours et, s'agissant des textes littéraires, de la littérature. (1998: 109)

On ne saurait mieux synthétiser en quelques lignes l'essentiel du programme herméneutique, qu'on reconnaît tant à la prise en charge par l'interprète de son propre regard, qu'au souci de désigner les conceptions spécifiques du langage, de la constitution du sens et du phénomène littéraire employées à l'exercice interprétatif. Ce qui distingue l'herméneutique d'autres modes d'approche du texte a probablement à voir avec la prééminence qu'elle accorde à certains concepts réflexifs, telle l' "historicité» qui consiste à marquer, et non à masquer, la situation à la fois déterminée et déterminante de l'interprète dans le dialogue qu'il tente d'établir avec l'objet esthétique, prémisse qui disqualifie d'emblée la tendance inverse visant précisément à dénier cette situation particulière de l'interprète pour "faire dépendre l'objectivité d'un effacement complet du sujet interprétant» que Jean Grondin (1993: 177) n’hésite pas à qualifier de «chimère de l'historicisme».

Si je privilégie de développer ce regard pour aborder des œuvres du passé ou du présent, je ne peux pas, dans cette perspective herméneutique, me déliter de ma propre situation historique; il me faudra au contraire l'inclure et la prendre en compte dans un rapport spécifique à l'objet. L'attitude esthétique que semblent 
faire valoir Gadamer et H.R. Jauss de l'École de Constance («attitude» de loin préférée à l'idée de «méthode») consiste à traiter avec les obscurités particulières de l'objet de langage dans une disposition interprétative dite interrogative. Une question est posée à l'objet, qui montre, en même temps que sa volonté de savoir, le lieu d'où est appelé ce savoir de l'autre: la deixis du sujet et ses circonstances d'énonciation particulières. Véritable pierre de touche du projet herméneutique, la dialectique de la question et de la réponse cherche à faire émerger la question de l'autre - de l'objet - au gré de la nécessaire mise au jour de la question du sujet qui s'y montre - l'instance énonciatrice -, en ouvrant du même coup un procès de signification où la médiation réciproque de l'objet et du sujet ne connaît pas de trêve, puisque que toute ouverture à l'autre se trouve aussi décloisonner quelque rapport à soi, l'herméneutique postulant que

[...] la compréhension renferme toujours une part de compréhension de soi, un Sichverstehen, une «application» à soi. Comprendre c'est «s'y» comprendre à quelque chose. Dans les termes de Gadamer, comprendre veut dire avoir réussi à appliquer un sens à notre situation, avoir trouvé une réponse à nos questions. (Grondin, 1993: 176)

La compréhension, d'après l'acception de Gadamer, en se présentant dans un double aspect transitif et intransitif, accuse le caractère réflexif et donne le branle au mouvement de l'interprétation.

Mais qu'en est-il de l'expérience esthétique et du problème de l'autoréflexivité en littérature? Comment établir une relation entre la "distanciation" prescrite par la forme même du texte autoréflexif - texte «limite» dont la réflexion sur lui-même se traduit souvent sous l'œil du lecteur par une apparente fermeture à l'autre - et l'expérience de lecture, qui peuvent d'emblée paraître inconciliables? Comment conjoindre deux tendances si éloignées l'une de l'autre, l'une procédant d'une mise à l'écart de soi devant la difficulté d'un objet qui tend lui-même à n'afficher que son propre retrait, et l'autre reposant sur la dimension jouissive qui participe de la rencontre de l'art? Ou, en d'autres termes encore, comment réunir dans la lecture une posture apollinienne - typique des sciences de l'esprit - et une disposition dionysiaque - propice au travail de l'imaginaire, à la créativité, et à l'inventivité formelles - et faire en sorte, si tension irréductible il y a entre les deux attitudes, qu'elle soit au moins productive pour le champ des études littéraires?

Une attention particulière s'attache ici à la dialectique sujet/objet, motivée tant par la préoccupation des œuvres de notre temps que par l'intérêt culturel, formel et esthétique qu'elles revêtent. Et une telle attention conduit à un souci non moins grand du sujet qui se constitue à leur contact - soit par la création ou par la lecture. L'occasion d'acquérir un savoir sur l'objet d'art ne favoriserait-elle pas la compréhension de la fonction que celui-ci exerce sur l'autre, notamment le sujet qu'il informe? Le recours à des concepts en chiasmes et autres paradoxismes est le passage obligé d'un propos qui cherche à rompre avec une certaine tradition dualiste héritée de l'idéologie de la modernité, dont il vise précisément à expérimenter la lézarde en laquelle, à un moment ou à un autre, le signe, objet ou sujet, dès lors qu'il est question d'une mise en langage et donc d'une communication à un tiers, peut risquer de se voir basculer en son contraire, et d'épouser le reflet inversé de l'autre. Les modalités de cette altérité, voire de cette "altérisation", restant toujours à dialectiser parce que dynamiques et protéiformes, se présentent comme inépuisables et engagent à tout le moins l'exercice de lecture à les saisir sur le même mode, c'est-à-dire avec la même mobilité, in situ.

\section{FraCtURE ET PLURALITÉ DE L'OBJET DE LANGAGE}

Si le langage lui-même ne repose pas tout entier sur un fabuleux paradoxe, il procède à tout le moins d'une dialectique: non seulement use-t-on, pour le qualifier, d'une profusion de vocables, tous recelant l'idée d'une "pluralité» en commun dénominateur, mais est-on aussi porté, à l'inverse, à le caractériser par un manque à son origine. 
Une kyrielle de paradoxes et de rapports dialectiques sont en mesure de rendre compte des nombreux phénomènes d'interface qui ont marqué la culture et la pensée du siècle et participé des coupures épistémologiques importantes dans les sciences humaines. Savoir, notamment, le langage, que des penseurs comme Roland Barthes nous ont amenés à considérer comme seule réalité, "puisqu'il n'y a rien en dehors du langage, mais qu'en outre ces langues, que nous croyons parler, en fait nous parlent” (Favre, 1990: 203-204). Or, il est convenu, de nos jours, de traiter l'art et la littérature comme actes de discours singuliers. Qu'il suffise seulement de rappeler, à cet effet, la théorie de Ducrot qui a rayé une fois pour toutes la vieille certitude d'un sujet unitaire (certitude déjà émoussée par les travaux de Bakhtine). Ducrot présente en effet l'énoncé comme une «description de l'énonciation en laquelle [se trouve] la caractéristique générale du sens» (1989: 190-191). C'est dire ici que l'énoncé - tout objectif qu'il puisse être - n'aurait de sens que dans la mesure où il surplomberait et réfléchirait l'énonciation (lieu d'inscription du sujet). C'est déjà la mise en exergue du caractère paradoxal du langage qui semble contenir en son principe même un dynamisme faisant alterner l'avancée (transitive) vers l'autre et le retour (intransitif) à soi.

Parce que la subjectivité ne se donne jamais, tout comme

l'objectivité du reste, son caractère insaisissable invite à ne porter attention qu'à ses modes de représentation et, ainsi, à se replier sur une pragmatique du sujet. (Larochelle, 1995: 34)

L'herméneutique gadamérienne ne fait pas exception non plus à cette conscience du pluriel au cœur du phénomène de la représentation. Gadamer (1996: 401) ne dira-t-il pas que c'est «à partir du dialogue que nous sommes, [que] nous cherchons à nous approcher de l'obscurité du langage»? L'herméneutique postule un dialogisme et une intersubjectivité inhérents à l'usage de la langue par le sujet et on pourrait par là l'affilier à une certaine conception dialectique de la modernité. Alors qu'un discours postmoderne suppose la pluralité sur fond de chaos à l'origine d'un relativisme et d'un nivellement étendu à toutes les sphères du discours, l'herméneutique procéderait plutôt de la reconnaissance d'une pluralité, certes, mais assistée d'une instance organisatrice (la conscience?), ces deux tendances (de transcendance dans l'immanence) se rencontrant dans ce que nous pourrions convenir d'appeler, d'après la formule qu'on attribue à Félix Torres, une métamodernité.

\section{FRACTURE ET PLURALITÉ DU SUJET}

La faiblesse et l'impuissance du sujet moderne à s'autoriser lui-même se voient confirmées par des formules définitives qui menacent son pouvoir. Pensons par exemple à celle-ci de Hermann Parret: «le sujet est davantage l'effet de l'énonciation que sa cause" (Martin et Panier, 1993: 22). Toute action humaine ayant dû jusqu'alors s'inféoder au règne de la totale maitrise des choses par l'esprit, commencent à se creuser, à l'ère du soupçon, une brèche, une faille qui altèrent désormais la belle assurance du logos, du cogito cartésien et le leurre de parfaite connivence de l'homme et de la nature auxquels des siècles de positivisme avaient voulu faire croire. De tout-puissant qu'il était, de mort qu'on l'a ensuite proclamé, il est là, le sujet contemporain, qui fait retour, mais sous une facture plus modeste. Modestie causée notamment par le récent détachement des origines divines que la modernité lui faisait prendre pour siennes. Un tel sujet accepte d'aller plus loin en lui-même tandis qu'il descend plus profondément dans son environnement extérieur, d'exhiber son imperfection et son incomplétude originaires, prêt à risquer la rencontre et à consentir à de nouvelles formes d'expériences langagières et esthétiques, et plus conscient, désormais, que la formation de son identité passe par un recours à l'autre. Lien à créer, à construire, qui opère dans la plus grande complexité dans l'expérience esthétique, quand on tente de comprendre le sens d'une ouvre qui semble défier tous les points de repère, ici l'objet de contemplation de Francis Jacques:

[...] moins l'autre est capable de me recevoir, plus j'ai besoin de le détruire ou de l'exclure; mais plus je l'exclus, plus je ressens mon vide et mon impuissance propres. Course infinie où jamais 
je ne m'atteins. Que je tente de supprimer l'autre pour acquérir la certitude de mon essence propre, je me mets à menacer ma propre identité. (Jacques, 1982: 80)

La question du sujet partageant avec le langage ce même élément d'ambiguitté irréductible, ou aporétique, ainsi que l'entendait Hannah Arendt, on voit qu'elle a partie liée avec l'approche allemande de la réception, particulièrement avec celle de l'expérience esthétique où il est question d'un effacement provisoire du sujet pour qu'il puisse se saisir, la «non-identification au phénomène observé n'[étant] pas un choix, mais un destin; une condition de la réflexivité» (Larochelle, 1995: 30). Effacement rendu possible par une dialectique avec l'objet (prosaïquement, dialogue ou dynamisme avec l'objet). Cette expérience esthétique de «jouissance de soi dans la jouissance de l'autre», concept à forte densité dialectique de H.R. Jauss, convoque l'idée d'une jouissance de soi-même, certes, mais qui n'aurait de narcissique que l'apparence, puisqu'elle s'est prêtée à l'exercice de l'objectivation dans une attitude de dépassement de soi-même et d'ouverture à l'autre (l'objet d'art, le texte littéraire). Ce rapport fait bien voir que plus radicale est l'expérience de l'étrangeté qu'un lecteur fait d'un texte, par exemple, «[...] plus [elle] demande un effort d'adaptation de sa part, et par conséquent plus elle contrôle son attention" (Riffaterre, 1994: 284). L'attitude à privilégier face à l'art et à la fiction ne peut faire autrement que procéder d'une réflexion sur soi, dénarcissisée, pourrait-on dire, parce qu'elle aurait part à un libre rapport à l'objet et donc aussi, de ce point de vue, parce qu'elle ne cristalliserait pas, demeurant au contraire toujours sujette à de successives transformations. La tendance dialectique suppose aussi, ce qu'on vient juste de voir, le travail en creux de la «négativité» (qui heurte aussi fortement le sens commun et le binarisme occidental). Cette rencontre avec l'objet (l'œuvre, le texte) opère dans la fusion des horizons irréductibles de deux temporalités en jouant de cette historicité dont on ne peut faire abstraction, mais à partir de laquelle l'expérience esthétique devient possible.

\section{OBJET ET SUJET DANS LE LANGAGE}

On peut bien sûr toujours croire constituer un sujet plein, mais puisque le sujet se constitue dans le langage, et puisqu'on reconnaît ce même langage inapte à tout exprimer, lui dont on vient pourtant de reconnaitre qu'il constitue en quelque sorte le point de départ du sujet, il faut donc dire que le langage produit un sujet lacunaire, en manque de l'autre pour prendre conscience de soi, et que ce n'est qu'au prix d'une relation à l'autre, et donc d'une transitivité nécessaire, qu'est offerte à ce sujet sa propre multiplicité, ou l'ondoiement multiple de sa personne, pour utiliser la belle expression de Jean Marcel. Relation à l'autre dont on ne saurait dire avec plus d'exactitude l'exigence de la fonder sur un langage commun:

Tout dialogue présuppose un langage commun, ou mieux: tout dialogue donne naissance à un langage commun. Il y a là, comme disent les Grecs, quelque chose qui a été déposé au milieu (de nous), quelque chose auquel les interlocuteurs ont part, et dont ils s'entretiennent. [...] Ce n'est pas là un processus extérieur, qui se bornerait à ajuster des outils; il n'est absolument pas exact de dire que les interlocuteurs s'adaptent l'un à l'autre; dans le dialogue réussi, ils se soumettent au contraire tous les deux à la vérité de la chose, et cette vérité les unit en une communauté nouvelle. L'explication-entente à laquelle on procède dans le dialogue ne consiste pas à faire tout simplement valoir et triompher son propre point de vue, elle est au contraire la métamorphose qui vise à introduire dans ce qui est commun, et à la faveur de laquelle nul ne reste ce qu'il était.

(Gadamer, 1996: 402)

La rencontre de l'autre appelle la reconnaissance de ses propres limites, notamment de ses limites spatiales, de son propre lieu déictique. Reconnaître les limites de son langage, c'est aussi un peu les repousser au sens où assumer quelque chose de l'ordre du manque revient toujours un peu à un comportement producteur.

Il incombe au langage de nommer cette expérience de la négativité du sujet et de faire état de l'appréhension du monde au gré de la conciliation des différents discours qui définissent le subjectum. Il est 
alors question d'une mise à distance de soi, propice à susciter une expérience dialectique de participation/ contemplation, work in progress où le sujet prend part au processus de négativité, au sens où un certain savoir sur soi et sur le monde n'est jamais stable ou acquis, mais lui-même provisoire en ce qu'il est toujours soumis à l'événement d'une nouvelle expérience susceptible de modifier, d'altérer cela même qu'il croyait inaliénable. La négativité, dialectique au regard de Gadamer, repose sur une dynamique suggérant un processus où le sujet serait non moins achevable que le sens. Parce que

[f]aire l'expérience d'un objet signifie n'avoir pas, jusqu'à présent, vu les choses correctement et savoir mieux désormais ce qu'il en est. La négativité de l'expérience a donc un sens particulièrement créateur. Loin de se réduire à une illusion que l'on perce à jour et par conséquent à une rectification, elle représente l'acquisition d'un savoir de vaste portée. Ce ne peut donc être d'un objet choisi que l'on fait expérience. Il doit au contraire être tel qu'il permette d'accéder à un savoir meilleur, non seulement de lui-même, mais de ce que l'on pensait savoir auparavant, c'est-à-dire d'un universel. La négation grâce à laquelle l'expérience atteint ce résultat est une négation déterminée. Nous qualifions de dialectique cette forme d'expérience. (Gadamer, 1996: 376)

Cette «métamorphose à la faveur de laquelle nul ne reste ce qu'il était» (ibid., p. 402) fait écho à «la jouissance de soi dans la jouissance de l'autre» de Jauss, en ce qu'il est fait nommément allusion au potentiel de renouvellement que porte en germe toute rencontre véritable, notamment celle avec l'œuvre d'art, potentiel qui affecte, travaille et transforme le sujet de la réception.

L'identité du sujet et du monde, du sujet et de son langage, de même que la compétence du langage à traduire le monde, à s'effacer face à lui pour mieux pouvoir en rendre compte, comme manifestations propres de l'idéal de clarté, d'unicité et de précision de cette épistémè, demeurent autant d'idéaux qui s'essoufflent. Mais commence à s'imposer de manière de plus en plus urgente, et avec de plus en plus d'acuité, l'idée selon laquelle l'homme doit se travailler, se moduler, se transformer pour accéder à meilleur statut de sujet dans le monde. S'il est vrai que le langage est la médiation du «je» et du monde, mieux : «un centre où le moi et le monde [...] se présentent dans leur mutuelle solidarité originelle» (ibid., p. 500), c'est qu'on est engagé en permanence dans le travail du cercle herméneutique, travail où toute partie est justiciable d'un certain voisinage de l'autre et où le monde se voit à son tour transformé par ses parties, le langage découvrant alors sa part éthique, porteuse de potentialités heuristiques pour le devenir du sujet dans le monde. Si le sujet et le monde se rencontrent dans le langage, celui-ci ne laisse plus de doute sur sa dimension intersubjective. L'œuvre réfléchissant sur elle-même m'enseigne que mon discours ne peut que subir l'influence de celui de l'autre, et que de m'y colleter peut infléchir mon rapport au monde.

C'est dans cette brèche instaurée par le doute que va précisément s'engager le courant postmoderne, s'activant, se développant et se générant à partir de cet état de doute généralisé, voire érigé en dogme, qui aura finalement donné lieu à une nouvelle rhétorique apparue en même temps qu'on a reconnu avec Lyotard l'hégémonie du langage qui, en dernière analyse, ne fait jamais que revenir et réfléchir sur luimême. Le langage allait devenir la médiation unique et suprême du sujet postmoderne, se dépouiller d'une transparence et d'une clarté illusoires pour favoriser la reconnaissance de significations plus évanescentes, de réalités plus subtiles et sibyllines, collaborer à l'instabilité des configurations du sens, sans cesse soumises à l'aléatoire et au spectre de la contradiction (hantise bien «moderne»), bref allait, par une manière de conscience rigoureuse de l'aléa et de l'arbitraire de tout signe (monde, sujet, objet, langage), évoquer l'inconsistance déterminante d'une nouvelle vision du monde désormais logée à l'enseigne de la fiction, de la déstabilisation des systèmes de sens et de l'exhibition des illusions.

Si certains prétendent que la modernité est bel et bien révolue (la renvoyant à l'esthétique des Lumières, l'Aufklärung), d'autres préfèrent la redéfinir en termes 
d'un idéal en quelque sorte toujours à atteindre (Alain Touraine, en sociologie, dans Critique de la modernité), d'autres encore, sans la rejeter entièrement, en retiendront plutôt certains aspects, afin de la prolonger, au lieu d'en proclamer la fin et, par conséquent, son entrée au temple de la postmodernité. Félix Torres désigne notamment cette modernité revisitée en utilisant le terme de «métamodernité». Méta, tout en supposant un «après", convoque l'idée d'un mouvement dépassant ou englobant l'objet dont il est question, indiquant à la fois une intégration et une mise à distance, une conscience critique de la modernité (une manière d'accommodation au sens de Piaget), au lieu de son rejet radical. Une manière aussi de contourner ce que Larochelle appelle les écueils du «relativisme et de la métaphysique» et de travailler, par conséquent, à la transformation de ce qui est.

En ce qui touche l'objet, on peut tenter de comprendre une postmodernité à travers les différentes formes de représentation, de réflexivité, ou de référence tant dans les discours que dans les différents espaces disciplinaires. Les formes artistiques et littéraires qui réfléchissent sur ellesmêmes ne sont rien d'autre que les parangons de cette culture, de ce hic et nunc postmodernes (si l'on se place du point de vue de l'histoire) qui cherchent à s'épuiser sur un plan formel. Il n'est plus rare pour le roman d'accuser, entre autres, son fonctionnement métalinguistique/métatextuel, de passer au crible sa propre matérialité langagière, d'indiquer son inaptitude à décrire le réel ou à représenter le sujet, de métisser les codes génériques en modulant le rapport de la fiction et du réel par l'autofiction, de procéder à l'immersion de la pensée dans la fiction et de la fiction dans la pensée. Il s'agit d'y aller de constructions et de déconstructions successives et d'autres procédés du même ordre: collage, fragmentation, hybridité, hétérogénéité, délinéarisation, dénarrativisation, pluralité, polysémie, polyphonie, et, tout à la fois, de s'inscrire en faux vis-à-vis d'absolus qui ne tiennent plus, de réfuter toute forme de transcendance, d'invalider les lois, sans pour autant avoir élu de fondements de substitution pour le sujet. Enfin, cette catégorie particulière de textes littéraires qui, non seulement disent mais montrent l'incertitude de l'énonciation, serait à mettre sur le compte des traits assez typiques d'une esthétique bien postmoderne, voire métamoderne, dont on peut s'étonner que la critique semble, au mieux, disposée à ne constater que la seule existence et non à en analyser aussi les effets sur les lecteurs. L'exercice même qui s'applique à la recension des jeux ou procédés textuels, de mouvements de retour du signe sur lui-même, notamment, pourrait ressortir à quelque type de lecture postmoderne alors qu'en revanche, une disposition à comprendre au plus près ce phénomène paradoxal, à en aborder l'incidence sur le sujet de la réception, pourrait désigner une tendance critique d'allégeance métamoderne à la Félix Torres et dont l'une des plus brillantes applications est peut-être Qui suis-je et qui es-tu? que Gadamer consacre à un recueil de poésies de Paul Celan. Mais encore faut-il, pour atteindre à cette justesse dans son rapport à l'objet, reconnaître (réflexivement) que l'on participe soi-même de l'élaboration du sens, dont l'événement est tributaire des efforts requis pour en rendre compte.

[...] C'est ainsi que la conscience qui fait l'expérience s'est retournée [...] c'està-dire qu'elle a fait retour [...] à elle-même. Qui fait une expérience a pris conscience de son expérience - il est un homme d'expérience, c'està-dire qu'il a acquis un horizon nonveau à l'intérieur duquel quelque chose peut devenir pour lui expérience. (Gadamer, 1996: 376-377)

On pourrait alors repérer trois types de manifestations artistiques issus de tels discours idéologiques et retenir, d'un type moderne, qu'il tendrait à expulser tout ce qui ne se soumet pas d'emblée à sa logique binaire et, partant, au principe du tiers exclu; d'un deuxième type, disons postmoderne dans son acception commune, qu'il compulse, accumule, thésaurise avec ludisme - et parfois avec le goût d'un certain morbide, les objets et les croyances de tous ordres, dans l'indistinction ou l'indifférenciation; et d'un troisième type, disons métamoderne, une certaine 
propension à la pause, à l'attitude critique, qui imposerait à l'incessant tournoiement des choses un temps d'arrêt, aménageant une aire de repos, tandis qu'il semblerait mieux disposé à s'imposer à lui-même, c'est-à-dire à son propre regard, une contemplation active des indécidables que tend à forclore le premier, et une prise sur les éléments que la témérité du deuxième aligne, juxtapose, collige, cumule.

À ces trois types de discours (esthétiques, idéologiques), on pourrait corréler trois attitudes non moins typiques de la critique, calquées sur leur objet. La tendance moderne qui éradique le paradoxe au titre de contradiction, postulant l'objectivité absolue et rejetant toute idée selon laquelle son propre regard pourrait imposer à l'objet sa configuration particulière. La tendance postmoderne qui cite, montre l'aspect ludique et accessoire du paradoxe, souscrivant à la subjectivité pure et tendant à mépriser, par conséquent, une réflexion critique qui participerait nécessairement d'une certaine distanciation (le rejet, voire la honte, de l'intellectuel est encore assez général, notamment chez nous); et l'autre, la métamoderne, qui choisirait délibérément de confronter le paradoxe, qui en prendrait acte, ne postulant l'existence ni d'une objectivité absolue ni d'une subjectivité pure, mais assumant au contraire qu'on se situe toujours un peu sur la ligne de crête qui les sépare, dans la charnière poreuse qui les réunit.

Par son traitement particulier du paradoxe lié à la condition humaine, au langage et à ses productions, la pensée de René Payant pourrait emblématiser avec profit une telle attitude métamoderne lorsque, en 1980, dans une communication sur l'enseignement de l'histoire de l'art, il avait défini le postmoderne comme le point de rencontre de deux discours esthétiques, de deux idéologies, le romantisme et le formalisme (ou modernisme), conservant dans sa définition un peu des deux de sorte qu'il produit, en un seul énoncé lapidaire, une apothéose de sens heurtant la rationalité cartésienne: l'art contemporain devenait, selon sa formule, «une pratique subjective qui réfère à elle-même», c'est-àdire la pratique d'un sujet qui serait esthétique en ceci, précisément, qu'elle saurait rendre compte d'une objectivation, ou d'une mise à distance critique. On tendrait alors à reconnaître, dans cette perspective, que toute lecture, toute écriture, tout langage seraient donc, dans une certaine mesure, propres et universels (parce que là encore à dialectiser). Tout devenant tributaire de la forme sous laquelle on choisirait d'inscrire ce propre, ce particulier: sur le mode de l'affirmation (c'est-à-dire avec l'assomption du voile que la subjectivité, avec ses préjugés, ses expériences, peut introduire et faire jouer entre soi et l'objet), affirmation consciente, comme dirait Merleau-Ponty, de cette part de «négativité qui réalise la langue en la détruisant». Notre époque montre clairement dans les formes littéraires qu'elle a portées au jour cette «négativité » en acte. Évidemment, le personnel peut encore s'inscrire, malgré lui, sur le mode du déni (c'est-à-dire de l'affirmation réitérée de son refus, qui traduit l'acharnement du langage à se croire absolument clair et univoque). Mais c'est davantage au nom du caractère non définitif du sens et de sa quête infinie de l'expression juste qu'il se laissera saisir.

[...] l'herméneutique peut cependant faire preuve de plus de sympathie vis-à-vis de la thèse derridienne. Car sa conception d'une vérité de participation, son insistance sur l'horizon sans fin du dialogue qui nous encercle témoignent aussi du caractère tragiquement inatteignable d'un sens définitif et chosifié, tel que suggéré par la métaphysique de la présence et l'approche du langage qu'elle commande. Ici encore, il faut invoquer l'idée d'un mot intérieur investissant le travail de la parole. Selon la conviction la plus intime de l'herméneutique, aucun mot ne réussira jamais à épuiser la tension interne de la parole, en termes derridiens, la différance entre le mot prononcé et ce qu'il veut dire. Le signe ou le mot que l'on entend ne doit jamais être pris pour la présence ultime du sens. Il n'est que renvoi, signe vers, l'acte de différer, si l'on veut, à un autre que lui, mais qu'aucune parole ne parviendra jamais à rattraper. Toute notre expérience langagière vit finalement de cette recherche, de la différance qui se creuse entre le mot et sa volonté de sens. À ce titre, l'épreuve de la différance, de l'insatisfaction essentielle de l'ordre des signes, est la plus herméneutique qui soit.

(Grondin, 1993: 221) 
Wittgenstein, lui-même philosophe du langage avant la lettre, après avoir mené jusqu'au bout son hypothèse de langage pur et référentiel, a fini par conclure, à l'instar de Gadamer, qu'il demeurerait toujours, dans la réalité contingente du monde, de l'informulable et de l'indéfinissable pour mettre le langage en échec (de même que le sujet qu'il est censé produire). En fin de parcours, on eût pu le dire converti à l'herméneutique, quand il optait pour la désignation, l'assomption par le langage, de cela même qui pouvait risquer d'ébranler l'explication et résister aux tentatives de description. On fait valoir, dans cette perspective, qu'un comportement critique ne s'attachant qu'aux seuls éléments ostensibles d'un objet d'art entraînerait non seulement une fausse compréhension du phénomène esthétique, mais aussi ce que Gadamer appelle «une déformation effective de la connaissance». En fait, pour être scientifique, il faudrait tenir compte de l'objet en tant qu'il est toujours configuré par le langage (cf. notamment Gaston Bachelard dans La Formation de la pensée scientifique) et, par conséquent, de l'entité que le langage fait toujours plus ou moins surgir - le sujet.

En outre, dans ses Leçons et conversations, Wittgenstein (1971: 155) reconnaît chez l'humain placé dans des situations limites propices à faire vaciller ses compétences langagières une tendance à «donner du front contre les bornes du langage» et assimile à l'éthique la part de résistance du langage qui presse le sujet de "changer son style de pensée» dans l'expérience qu'il fait de sa propre finitude.

Dans un entretien récent, on a demandé à Gadamer ce qu'il tenait pour le plus digne d'être communiqué de son expérience de la vie. Il a répondu: "Je crois que ce qu'il y a de plus digne d'être communiqué, c'est ce qui ne se laisse pas communiquer».

(Grondin, 1993: 184)

\section{RÉFÉREN CES BIBLIO G RAPHIQ U ES}

ADORNO, T. W. [1991]: Minima moralia. Réflexions sur la vie mutilée, Paris, Payot, coll. «Critique de la politique».

ARENDT, H. [1972]: La Crise de la culture: huit exercices de pensée politique, Paris, Gallimard.

BACHELARD, G. [1967]: La Formation de l'esprit scientifique: contribution à une psychanalyse de la connaissance objective, Paris, Vrin, coll.

"Bibliothèque des textes philosophiques".

CARDINAL, J. [1998] : «Le même et l'autre. Un nouveau paradigme critique de l'analyse littéraire", Protée, vol. 26, n² (automne).

DUCROT, O. [1989]: Logique, structure, énonciation. Lectures sur le langage,

Paris, Minuit, coll. "Propositions".

FAVRE, R. (dir.) [1990] : La Littérature française. Histoire et perspectives, Lyon, Presses universitaires de Lyon.

GADAMER, H.-G. [1996]: Vérité et méthode. Les grandes lignes d'une herméneutique philosophique, Paris, Gallimard, coll. «L'ordre

philosophique";

[1987]: Qui suis-je et qui es-tu? Commentaire de Cristaux de souffle de Paul Celan, Arles, Actes Sud.

GENINASCA, J. [1998] : «Le discours n'est pas toujours ce que l'on croit", Protée, vol. 26, n 1.

GRONDIN, J. [1993]: L'Universalité de l'herméneutique, Paris, P.U.F., coll. "Épiméthée».

HochmanN, J. [1988] : «Rêverie sur les rapports du trompe-l'œil et de l'illusion, du fétiche et de l'objet transitionnel ", dans R. Court, L'Effet trompe-l'oeil dans l'art et la psychanalyse, Paris, Bordas/Dunod, 120.

JACQUES, F. [1982]: Différence et Subjectivité, Paris, Aubier Montaigne, coll. «Analyse de la raison".

JAUSS, H. R. [1988] : Pour une herméneutique littéraire, Paris, Gallimard, coll. «nrf».

LAROCHelle, G. [1995]: Philosophie de l'idéologie. Théorie de l'intersubjectivité, Paris, P.U.F., coll. «L'interrogation philosophique». MARCEL, J. [1996] : Fractions 1, Montréal, L'Hexagone, coll.

«Itinéraires/Carnets».

Martin, F. et L. PANier [1993]: «Figures et énonciation», Protée, vol. $21, \mathrm{n}^{\circ} 2$.

Merleau-Ponty, M. [1985] : L'Eil et l'Esprit, Paris, Gallimard, coll.

"Folio/Essais".

PAYANT, R. [1987]: Pièces détachées sur l'art 1976-1987, Laval, Trois, coll. "Vedute".

RifFAterRe, M. [1994]: «L'inscription du sujet», dans G. Molinié et P. Cahné (dir.), Qu'est-ce que le style?, Paris, P.U.F., coll. «Linguistique nouvelle".

TOURAINE, A. [1993]: Critique de la modernité, Paris, Fayard. WitTGENSTEIN, L. [1971]: Leçons et conversations. Suivies de Conférence sur l'Éthique, Paris, Gallimard, coll. «nrf». 